\title{
Remarks on hyperstability of the Cauchy functional equation
}

\author{
JANUSZ BRZDȨK
}

\begin{abstract}
We present some simple observations on hyperstability for the Cauchy equation on a restricted domain. Namely, we show that (under some weak natural assumptions) functions that satisfy the equation approximately (in some sense), must be actually solutions to it. In this way we demonstrate in particular that hyperstability is not a very exceptional phenomenon as it has been considered so far. We also provide some simple examples of applications of those results.
\end{abstract}

Mathematics Subject Classification (2010). Primary 39B82, 47H14, 47J20; Secondary 39B62.

Keywords. Cauchy equation, hyperstability, cocycle, Hyers-Ulam stability.

\section{Introduction}

In this paper $\mathbb{N}, \mathbb{Z}, \mathbb{Q}, \mathbb{R}$ and $\mathbb{C}$ denote the sets of positive integers, integers, rationals, reals and complex numbers, respectively; $\mathbb{N}_{0}:=\mathbb{N} \cup\{0\}$ and $\mathbb{R}_{+}:=[0, \infty)$. Moreover, $X$ and $Y$ always stand for normed spaces (unless clearly stated otherwise) and $U \subset X$ is nonempty.

In what follows we say that a function $f$ mapping $U$ into a set $Z$, endowed with a binary operation $+: Z^{2} \rightarrow Z$, is additive on $U$ provided it satisfies the conditional Cauchy functional equation

$$
f(x+y)=f(x)+f(y) \quad x, y \in U, x+y \in U ;
$$

if $U=X$, then we simply say that $f$ is additive.

We present some simple hyperstability results for Eq. (1.1). Namely, we show that, for some natural particular forms of $\varphi$ (and under some additional assumptions on $U$ ), the conditional functional Eq. (1.1) is $\varphi$-hyperstable in the class of functions $f: U \rightarrow Y$, i.e., each $f: U \rightarrow Y$ satisfying the inequality

$$
\|f(x+y)-f(x)-f(y)\| \leq \varphi(x, y) \quad x, y \in U, x+y \in U,
$$


must be additive on $U$. In this way we expect to stimulate somewhat the further research of the issue of hyperstability, which seems to be a very promising subject to study within the theory of Hyers-Ulam stability.

Let us recall that the problem of stability of functional equations was motivated by a question of S.M. Ulam asked in 1940 and an answer to it published by Hyers [22]. Since then numerous papers on this subject have been published and we refer to $[8-10,19,23,26,27,30,31]$ for more details, some discussions, further references and examples of very recent results. According to our best knowledge, the first hyperstability result was published in [4] and concerned ring homomorphisms. However, it seems that the term hyperstability was used for the first time in [29] (quite often it is confused with superstability, which admits also bounded functions).

\section{The first observations}

We start with the following three simple propositions for $U=X$.

Proposition 2.1. Let $(X,\langle\cdot \mid \cdot\rangle)$ be a real inner product space with $\operatorname{dim} X>1$ and $g: X \rightarrow Y$. Suppose that there are positive real numbers $p$ and $L$ with

$$
\|g(x+y)-g(x)-g(y)\| \leq L|\langle x \mid y\rangle|^{p} \quad x, y \in X .
$$

Then the following two statements are valid.

(A) If $p \neq 1$, then $g$ is additive.

(B) If $p=1$, then there exist additive $a: X \rightarrow Y$ and a vector $z_{0} \in Y$ such that $2\left\|z_{0}\right\| \leq L$ and

$$
g(x)=a(x)+\|x\|^{2} z_{0} \quad x \in X .
$$

Proof. Let $g_{1}$ and $g_{2}$ denote the odd and even parts of $g$, i.e.,

$$
g_{1}(x):=\frac{1}{2}(g(x)-g(-x)), \quad g_{2}(x):=\frac{1}{2}(g(x)+g(-x)), \quad x \in X .
$$

Then it is easily seen that

$$
\left\|g_{i}(x+y)-g_{i}(x)-g_{i}(y)\right\| \leq L|\langle x \mid y\rangle|^{p} \quad x, y \in X, i=1,2,
$$

which yields

$$
g_{i}(x+y)=g_{i}(x)+g_{i}(y) \quad x, y \in X,\langle x \mid y\rangle=0, i=1,2 .
$$

Hence, by [35, Theorem 5], $g_{1}$ is additive. Further, according to [35, Theorem $9]$, there exists an additive $b: \mathbb{R} \rightarrow Y$ such that $g_{2}(x)=b\left(\|x\|^{2}\right)$ for $x \in X$. Take $x_{0} \in X$ with $\left\|x_{0}\right\|=1$. Clearly, (2.2) with $x=y=\xi x_{0}$ implies that

$$
\left.2\left\|b\left(\xi^{2}\right)\right\|=\left\|b\left(\left\|2 \xi x_{0}\right\|^{2}\right)-2 b\left(\| \xi x_{0}\right)\right\|^{2}\right) \| \leq L \xi^{2 p} \quad \xi \in \mathbb{R},
$$


whence $b$ is linear. This means that $b(\xi)=\xi z_{0}$ for $\xi \in \mathbb{R}$ with $z_{0}:=b(1)$ and consequently

$$
g_{2}(x)=\|x\|^{2} z_{0} \quad x \in X .
$$

Now using (2.2) (with $x$ and $y$ replaced by $\xi x$ ) we get

$$
2 \xi^{2}\|x\|^{2}\left\|z_{0}\right\| \leq L \xi^{2 p}\|x\|^{2 p} \quad x \in X, \xi \in \mathbb{R},
$$

which is possible only when $p=1$ or $z_{0}=0$. Clearly, if $p=1$, then (2.3) yields that $2\left\|z_{0}\right\| \leq L$.

It is easily seen that if $g$ has the form described either by $(A)$ or by $(B)$, then it fulfils (2.1).

Proposition 2.2. Let $\operatorname{dim} X>2$ and $g: X \rightarrow Y$. Suppose that there are positive real numbers $p$ and $L_{0}$ with

$$
\|g(x+y)-g(x)-g(y)\| \leq L_{0}\left|\|x+y\|^{2}-\|x-y\|^{2}\right|^{p} \quad x, y \in X .
$$

Then the following two statements are valid.

( $\alpha$ ) If $p \neq 1$ or $X$ is not a real inner product space, then $g$ is additive.

( $\beta)$ If $X$ is a real inner product space and $p=1$, then there exist an additive mapping $a: X \rightarrow Y$ and a vector $z_{0} \in Y$ such that $\left\|z_{0}\right\| \leq 2 L_{0}$ and

$$
g(x)=a(x)+\|x\|^{2} z_{0} \quad x \in X .
$$

Proof. Note that (2.4) yields

$$
g(x+y)=g(x)+g(y) \quad x, y \in X,\|x+y\|=\|x-y\| .
$$

If $X$ is not a real inner product space, then it follows from [39] that the even part of $g$ is identically equal zero. This means that $g$ is odd and consequently it is additive in view of [38, Theorem, p. 270].

So it remains to consider the case where the norm in $X$ comes from some real inner product $\langle\cdot \mid \cdot\rangle$. Then (2.4) takes form (2.1) with $L=4 L_{0}$ and it is enough to use Proposition 2.1.

Proposition 2.3. Let $\operatorname{dim} X>2$ and let $g: X \rightarrow Y$. Suppose that there are functions $\eta, \mu: \mathbb{R} \rightarrow \mathbb{R}$ with $\mu(0)=0$ and

$$
\|g(x+y)-g(x)-g(y)\| \leq \mu(\eta(\|x\|)-\eta(\|y\|)) \quad x, y \in X .
$$

Then $g$ is additive.

Proof. Taking $x=y$ in (2.6) we obtain that

$$
g(x+y)=g(x)+g(y) \quad x, y \in X,\|x\|=\|y\| .
$$

Hence, by [38, Theorem 3.1], $g$ is additive. 


\section{Some further hyperstability results}

Given $A, B: X \rightarrow X$, for the simplicity of notations we write $A B:=A \circ B$ and define the mapping $A+B: X \rightarrow X$ by $(A+B)(x):=A(x)+B(x)$ for $x \in X$; moreover, if $V \subset X, U \subset V$ and $A: V \rightarrow X$, then

$$
\|A\|_{U}:=\inf \{\xi \in \mathbb{R}:\|A(x)-A(y)\| \leq \xi\|x-y\| \text { for } x, y \in U\} .
$$

It is easily seen that, in the particular case where $A$ is additive (i.e., $A(x+y)=$ $A(x)+A(y)$ for every $x, y \in X$ ), we have (with $U=X$ )

$$
\|A\|_{X}=\inf \{\xi \in \mathbb{R}:\|A(x)\| \leq \xi\|x\| \text { for } x \in X\} .
$$

Now, we are in a position to show the following result.

Theorem 3.1. Assume that $C, D: X \rightarrow X$ are additive,

$$
\begin{gathered}
C D=D C, \\
C(x), D(x), C(x)+D(x) \in U \quad x \in U .
\end{gathered}
$$

Let $p \in \mathbb{R}_{+}$be such that one of the following two conditions is valid:

(a) $E:=D+C$ is injective, $U \subset E(U)$ and

$$
\left(\|D\|_{U}^{p}+\|C\|_{U}^{p}\right)\left\|E^{-1}\right\|_{U}^{p}<1 ;
$$

(b) $U \subset D(U), D$ is injective and

$$
\left(\|E\|_{U}^{p}+\|C\|_{U}^{p}\right)\left\|D^{-1}\right\|_{U}^{p}<1 .
$$

Then every function $g: U \rightarrow Y$ for which there exists $L \in \mathbb{R}_{+}$such that

$$
\|g(x+y)-g(x)-g(y)\| \leq L\|C(x)-D(y)\|^{p} \quad x, y \in U, x+y \in U,
$$

is additive on $U$.

Proof. In view of (3.2), from (3.3) (with $x$ replaced by $D(x)$ and $y=C(x)$ ) we obtain

$$
g((D+C) x)=g(D(x))+g(C(x)) \quad x \in U .
$$

First consider the case of (a). Then $U=E(U)$ and (3.4) yields

$$
g(x)=g\left(D E^{-1} x\right)+g\left(C E^{-1} x\right) \quad x \in U .
$$

Let $\kappa:=\left(\|D\|_{U}^{p}+\|C\|_{U}^{p}\right)\left\|E^{-1}\right\|_{U}^{p}$. We show that, for each $n \in \mathbb{N}_{0}$,

$$
\begin{array}{r}
\|g(x+y)-g(x)-g(y)\| \leq \kappa^{n} L\|C(x)-D(y)\|^{p} \\
x, y \in U, x+y \in U .
\end{array}
$$


The proof is by induction. Clearly the case $n=0$ is just (3.3). So fix $l \in \mathbb{N}_{0}$ and assume that (3.6) holds true with $n=l$. Then, by (3.5),

$$
\begin{aligned}
&\|g(x+y)-g(x)-g(y)\| \\
&= \| g\left(D E^{-1}(x+y)\right)+g\left(C E^{-1}(x+y)\right)-g\left(D E^{-1}(x)\right) \\
& \quad-g\left(C E^{-1}(x)\right)-g\left(D E^{-1}(y)\right)-g\left(C E^{-1}(y)\right) \| \\
& \leq\left\|g\left(D E^{-1}(x)+D E^{-1}(y)\right)-g\left(D E^{-1}(x)\right)-g\left(D E^{-1}(y)\right)\right\| \\
&+\left\|g\left(C E^{-1}(x)+C E^{-1}(y)\right)-g\left(C E^{-1}(x)\right)-g\left(C E^{-1}(y)\right)\right\| \\
& \leq \kappa^{l} L\left(\left\|C D E^{-1}(x)-D D E^{-1}(y)\right\|^{p}\right) \\
&+\kappa^{l} L\left\|C C E^{-1}(x)-D C E^{-1}(y)\right\|^{p} \quad x, y \in U, x+y \in U .
\end{aligned}
$$

Since, according to (3.1), $C E^{-1}(x)=E^{-1} C(x)$ and $D E^{-1}(x)=E^{-1} D(x)$ for each $x \in U$, this means that

$$
\begin{aligned}
&\|g(x+y)-g(x)-g(y)\| \\
& \leq \kappa^{l} L\left(\left\|D E^{-1} C(x)-D E^{-1} D(y)\right\|^{p}\right) \\
&+\kappa^{l} L\left\|C E^{-1} C(x)-C E^{-1} D(y)\right\|^{p} \\
& \leq \kappa^{l} L\left(\|D\|_{U}^{p}\left\|E^{-1}\right\|_{U}^{p}+\|C\|_{U}^{p}\left\|E^{-1}\right\|_{U}^{p}\right)\|C(x)-D(y)\|^{p} \\
&= \kappa^{l+1} L\|C(x)-D(y)\|^{p} \quad x, y \in U, x+y \in U .
\end{aligned}
$$

Thus we have proved that (3.6) is valid for each $n \in \mathbb{N}_{0}$. Since $\kappa<1$, letting $n \rightarrow \infty$ in (3.6) we obtain that $g$ is additive on $U$.

Next assume that (b) holds. From (3.4) we deduce that

$$
g(x)=g\left(E D^{-1}(x)\right)-g\left(C D^{-1}(x)\right) \quad x \in U .
$$

Write $\eta:=\left(\|E\|_{U}^{p}+\|C\|_{U}^{p}\right)\left\|D^{-1}\right\|_{U}^{p}$. We show by induction that, for each $n \in \mathbb{N}_{0}$,

$$
\begin{array}{r}
\|g(x+y)-g(x)-g(y)\| \leq \eta^{n} L\|C(x)-D(y)\|^{p} \\
x, y \in U, x+y \in U .
\end{array}
$$

The case $n=0$ is trivial. Take $l \in \mathbb{N}_{0}$ and assume that (3.8) is valid for $n=l$. Then, by (3.7),

$$
\begin{aligned}
& \|g(x+y)-g(x)-g(y)\| \\
& =\| g\left(E D^{-1}(x+y)\right)-g\left(C D^{-1}(x+y)\right)-g\left(E D^{-1}(x)\right) \\
& \quad+g\left(C D^{-1}(x)\right)-g\left(E D^{-1}(y)\right)+g\left(C D^{-1}(y)\right) \| \\
& \leq \\
& \quad\left\|g\left(E D^{-1}(x)+E D^{-1}(y)\right)-g\left(E D^{-1}(x)\right)-g\left(E D^{-1}(y)\right)\right\| \\
& \quad+\left\|g\left(C D^{-1}(x)+C D^{-1}(y)\right)-g\left(C D^{-1}(x)\right)-g\left(C D^{-1}(y)\right)\right\|
\end{aligned}
$$




$$
\begin{aligned}
\leq & \eta^{l} L\left\|C E D^{-1}(x)-D E D^{-1}(y)\right\|^{p} \\
& +\eta^{l} L\left\|C C D^{-1}(x)-D C D^{-1}(y)\right\|^{p} \\
= & \eta^{l} L\left(\|E\|_{U^{p}}\left\|D^{-1}\right\|_{U}^{p}+\|C\|_{U}^{p}\left\|D^{-1}\right\|_{U}^{p}\right)\|C(x)-D(y)\|^{p} \\
= & \eta^{l+1} L\|C(x)-D(y)\|^{p} \quad x, y \in U, x+y \in U .
\end{aligned}
$$

Thus we have proved by induction that (3.8) is valid for each $n \in \mathbb{N}_{0}$. Since $\eta<1$, letting $n \rightarrow \infty$ in (3.8) we obtain that $g$ is additive on $U$.

Remark 3.2. Observe that condition (3.1) in Theorem 3.1 is valid for instance when $D=C^{n}$ with some $n \in \mathbb{N}_{0}$ or $D x=\gamma x$ for $x \in X$ with some $\gamma \in \mathbb{Q}$ (because $C$ is assumed to be additive).

Remark 3.3. Note that the inequality in (a) holds when $p>1, U=X$ and $C(x)=D(x)=\lambda x$ for $x \in X$, with some $\lambda \in \mathbb{R}$. Analogously, the inequality in (b) holds when $p>1, U=X, C(x)=-\lambda x$ and $D(x)=2 \lambda x$ for $x \in X$ (with some $\lambda \in \mathbb{R}$ ). It is easy to find several further examples.

There arises a natural open problem whether we can get similar hyperstability results in some of the situations where neither condition (a) nor (b) is fulfilled. In some of these cases we can derive some complementary stability and hyperstability results from the subsequent proposition, which follows easily from [7, Theorem 1] (cf. also [21]).

Proposition 3.4. Let $V \subset X$ be nonempty, $\varphi: V^{2} \rightarrow \mathbb{R}$ and $f: V \rightarrow Y$ satisfy

$$
\|g(x+y)-g(x)-g(y)\| \leq \varphi(x, y) \quad x, y \in V, x+y \in V .
$$

Suppose that $Y$ is complete and there is $\varepsilon \in\{-1,1\}$ such that $2^{\varepsilon} V \subset V$ and

$$
\begin{aligned}
& H(x):=\sum_{i=0}^{\infty} 2^{-i \varepsilon} \varphi\left(2^{i \varepsilon} x, 2^{i \varepsilon} x\right)<\infty \quad x \in V, \\
& \liminf _{n \rightarrow \infty}\left|2^{-n \varepsilon} \varphi\left(2^{n \varepsilon} x, 2^{n \varepsilon} y\right)\right|=0 \quad x, y \in V .
\end{aligned}
$$

Then there exists a unique $F: V \rightarrow Y$ that is additive on $V$ and

$$
\|F(x)-f(x)\| \leq H_{0}(x) \quad x \in V,
$$

where

$$
H_{0}(x):= \begin{cases}2^{-1} H(x), & \text { if } \varepsilon=1 ; \\ H\left(2^{-1} x\right), & \text { if } \varepsilon=-1 .\end{cases}
$$

Proposition 3.4 yields in particular the subsequent two corollaries generalizing the results of Hyers [22], Aoki [2], Rassias [32,33] and Gajda [20] (see also [34, Theorem 1]). 
Corollary 3.5. Let $Y$ be complete, $g: U \rightarrow Y, \delta, L_{1}, L_{2} \in \mathbb{R}_{+}, q, r \in(-\infty, 1)$, and $2 U \subset U$. Suppose that there exist $L \in \mathbb{R}_{+}, p \in(0,1)$, and $C: U \rightarrow X$ such that

$$
\|C(2 x)-C(2 y)\| \leq 2\|C(x)-C(y)\| \quad x, y \in U
$$

and

$$
\|g(x+y)-g(x)-g(y)\| \leq \delta+L_{1}\|x\|^{q}+L_{2}\|y\|^{r}+L\|C(x)-C(y)\|^{p}
$$

for every $x, y \in U \backslash\{0\}$ with $x+y \in U \backslash\{0\}$. Then there exists a unique function $G: U \rightarrow Y$ that is additive on $U$ and satisfies

$$
\|G(x)-g(x)\| \leq \delta+\frac{L_{1}\|x\|^{q}}{2-2^{q}}+\frac{L_{2}\|y\|^{r}}{2-2^{r}} \quad x \in U .
$$

Proof. It is enough to use Proposition 3.4 with $V:=U \backslash\{0\}, \varepsilon=1$ and

$$
\varphi(x, y):=\delta+L_{1}\|x\|^{q}+L_{2}\|y\|^{r}+L\|C(x)-C(y)\|^{p} \quad x, y \in U
$$

and next take $G(x):=F(x)$ for $x \in V$. Further, in the case when $0 \in U$, we must have $G(0)=0$. Since in such a situation

$$
\|g(0)\| \leq \delta
$$

$G$ defined in this way fulfils (3.10).

Corollary 3.6. Let $Y$ be complete, $g: U \rightarrow Y, L_{1}, L_{2} \in \mathbb{R}_{+}, q, r \in(1, \infty)$, and $U \subset 2 U$. Suppose that there exist $L \in \mathbb{R}_{+}, p \in(1, \infty)$, and $C: U \rightarrow X$ such that

$$
\left\|C\left(\frac{1}{2} x\right)-C\left(\frac{1}{2} y\right)\right\| \leq \frac{1}{2}\|C(x)-C(y)\|
$$

and

$$
\|g(x+y)-g(x)-g(y)\| \leq L_{1}\|x\|^{q}+L_{2}\|y\|^{r}+L\|C(x)-C(y)\|^{p}
$$

for every $x, y \in U$ with $x+y \in U$. Then there exists a unique function $G$ : $U \rightarrow Y$ that is additive on $U$ and satisfies

$$
\|G(x)-g(x)\| \leq \frac{L_{1}\|x\|^{q}}{2^{q}-2}+\frac{L_{2}\|y\|^{r}}{2^{r}-2} \quad x \in U .
$$

Proof. It is enough to use Proposition 3.4 with $V:=U, \varepsilon=-1$ and

$$
\varphi(x, y):=L_{1}\|x\|^{q}+L_{2}\|y\|^{r}+L\|C(x)-C(y)\|^{p} \quad x, y \in U .
$$

Note that Corollaries 3.5 and 3.6 with $\delta=L_{1}=L_{2}=0$ supply additional two hyperstability results, which cannot be deduced from Theorem 3.1. 
Remark 3.7. In connection with the statements of Theorem 3.1 and Corollaries 3.5 and 3.6 there arises the natural question when a function that is additive on $U$ can be extended to an additive function $f: X \rightarrow Y$. Some information on investigations of this issue can be found in [1], [28, Theorem 1.1, Ch. XVIII]) and [36, Chapter 4] (see also [37, pp. 143-4] for some extension procedure). Below we provide one more result concerning this problem, which corresponds somewhat to the outcomes in [1]. (Let us recall that $\mathcal{I} \subset 2^{X}$ is an ideal provided $A \cup B \in \mathcal{I}$ and $2^{A} \subset \mathcal{I}$ for every $\left.A, B \in \mathcal{I}\right)$.

Proposition 3.8. Let $h: U \rightarrow Y$ satisfy

$$
h(x+y)=h(x)+h(y) \quad x, y \in U, x+y \in U .
$$

Assume that there exists an ideal $\mathcal{I} \subset 2^{X}$ such that $X \notin \mathcal{I}, X \backslash U \in \mathcal{I}$ and

$$
B+x \in \mathcal{I} \quad B \in \mathcal{I}, x \in X .
$$

Then there is a unique additive $f: X \rightarrow Y$ such that $h(x)=f(x)$ for $x \in U$.

Proof. It is easy to deduce from [6, Lemma 1] that

$$
U-U:=\{x-y: x, y \in U\}=X .
$$

Take $a, b, c, d \in U$ with $a-b=c-d$ and write

$$
U_{1}:=(U-a) \cap(U-a-d), \quad U_{2}:=(U-b) \cap(U-b-c) .
$$

Clearly, by (3.12), $X \backslash U_{1}, X \backslash U_{2} \in \mathcal{I}$, whence $U_{0}:=U \cap U_{1} \cap U_{2} \neq \emptyset$.

Let $v \in U_{0}$. Then $v, v+a, v+b, v+a+d, v+b+c \in U$. Consequently, by $(3.11)$

$$
\begin{aligned}
h(v)+h(b)+h(c) & =h(v+b)+h(c)=h(v+b+c) \\
& =h(v+a+d)=h(v+a)+h(d) \\
& =h(v)+h(a)+h(d) .
\end{aligned}
$$

Thus we have proved that

$$
h(a)-h(b)=h(c)-h(d) \quad a, b, c, d \in U, a-b=c-d .
$$

According to (3.13), we may define $f: X \rightarrow Y$ by:

$$
f(z)=h(a)-h(b) \quad z \in X, a, b \in U, z=a-b .
$$

First we show that $f$ is an extension of $h$. To this end fix $z \in U$ and $u \in U \cap(U-z)$. Then (3.11) yields

$$
f(z)=f(z+u-u)=h(z+u)-h(u)=h(z)+h(u)-h(u)=h(z) .
$$

Next we prove that $f$ is additive. Take $z, w \in X$. According to (3.13), $z=a-b$ and $w=c-d$ for some $a, b, c, d \in U$. Hence $f(z)=h(a)-h(b)$ and $f(w)=h(c)-h(d)$. Take

$$
u \in U \cap(U-a) \cap(U-a-c) \cap(U-b) \cap(U-b-d) .
$$


Then

$$
\begin{aligned}
f(z+w) & =f(u+a+c-(u+b+d))=h(u+a+c)-h(u+b+d) \\
& =h(u+a)+h(c)-(h(u+b)+h(d)) \\
& =h(u)+h(a)+h(c)-(h(u)+h(b)+h(d)) \\
& =h(a)-h(b)+h(c)-h(d)=f(z)+f(w) .
\end{aligned}
$$

To complete the proof it remains to show the uniqueness of $f$. So, suppose that $f_{1}: X \rightarrow Y$ is additive and $f_{1}(x)=h(x)$ for $x \in U$. Take $z \in X$ and $a, b \in U$ with $z=a-b$. Then

$$
f_{1}(z)=f_{1}(a-b)=f_{1}(a)-f_{1}(b)=h(a)-h(b)=f(a-b)=f(z) .
$$

Remark 3.9. Below we give some natural examples of ideals $\mathcal{I}$ satisfying condition (3.12).

(a) $\mathcal{I}$ is the family of all subsets $T$ of $X$ with $\operatorname{card} T<\operatorname{card} X$.

(b) $\mathcal{I}$ is the family of all bounded subsets of $X$.

(c) $\mathcal{I}$ is the family of all first category subsets of $X$.

(d) $X=\mathbb{R}^{n}$ with some $n \in \mathbb{N}$ and $\mathcal{I}$ is the family of all subsets of $X$ that are of finite Lebesgue measure.

(e) $X$ is a Polish space and $\mathcal{I}$ is the $\sigma$-ideal of Christensen zero subsets of $X$ (see, e.g., [18]).

\section{Some consequences}

In what follows, given $\mathcal{I} \subset 2^{X}$ and $f, g: X \rightarrow Y$, we say that $f=g \mathcal{I}$-almost everywhere (abbreviated to $\mathcal{I}$-a.e.) in $X$ if there is a set $T \in \mathcal{I}$ such that $f(x)=g(x)$ for every $x \in X \backslash T$. Moreover we write $\alpha T:=\{\alpha x: x \in T\}$ for $T \subset X$ and $\alpha \in \mathbb{R}$. The next theorem is a consequence of some previous results in this paper. (An ideal $\mathcal{I} \subset 2^{X}$ is a $\sigma$-ideal provided $\bigcup_{n \in \mathbb{N}} T_{n} \in \mathcal{I}$ for every family of sets $\left.\left\{T_{n}\right\}_{n \in \mathbb{N}} \subset \mathcal{I}\right)$.

Theorem 4.1. Let $g: X \rightarrow Y$ and $\mathcal{I} \subset 2^{X}$ be a $\sigma$-ideal such that (3.12) holds and

$$
\alpha T \in \mathcal{I} \quad T \in \mathcal{I}, \alpha \in \mathbb{R} .
$$

Assume that one of the following two conditions is fulfilled.

(i) There exist $T \in \mathcal{I}, c, d \in \mathbb{R}, c d(c+d) \neq 0$, and reals $L>0$ and $p>1$ such that

$$
\|g(x+y)-g(x)-g(y)\| \leq L\|c x-d y\|^{p} \quad x, y \in X \backslash T .
$$

(ii) There exist $T \in \mathcal{I}, C: X \rightarrow X$ with $C(2 x)=2 C(x)$ for $x \in X$, and positive reals $L$ and $p \neq 1$ such that 


$$
\|g(x+y)-g(x)-g(y)\| \leq L\|C(x)-C(y)\|^{p} \quad x, y \in X \backslash T .
$$

Then there is a unique additive operator $f: X \rightarrow Y$ with $f=g \mathcal{I}$-a.e. in $X$.

Proof. First assume that (i) holds. Define $C, D: X \rightarrow X$ by: $C(x)=c x$ and $D(x)=d x$ for $x \in X$. Write $X_{T}:=X \backslash T$,

$$
U_{1}:=\bigcap_{n \in \mathbb{Z}}(c+d)^{n} X_{T}, \quad U_{2}:=\bigcap_{n \in \mathbb{Z}} c^{n} X_{T}, \quad U_{3}:=\bigcap_{n \in \mathbb{Z}} d^{n} X_{T},
$$

and $U:=U_{1} \cap U_{2} \cap U_{3}$. It is easily seen that $X \backslash U \in \mathcal{I}, c U=U, d U=U$ and $(c+d) U=U$. Further, if $c d>0$, then $|c+d|=|c|+|d|$ and consequently

$$
|d|^{p}+|c|^{p}<|c+d|^{p}
$$

if $c d<0$, then (without loss of generality, because (4.2) is symmetric with regard to $x$ and $y$ ) we can assume that $|d|=|c|+|d+c|$ and consequently

$$
|d+c|^{p}+|c|^{p}<|d|^{p} \text {. }
$$

This means that one of conditions (a) and (b) of Theorem 3.1 is valid and consequently $g$ is additive on $U$. Hence, by Proposition 3.8, there is an additive operator $f: X \rightarrow Y$ with $g(x)=f(x)$ for $x \in U$. The uniqueness of $f$ also follows from Proposition 3.8.

If (ii) holds, then we write

$$
U:=\bigcap_{n \in \mathbb{Z}} 2^{n}(X \backslash T)
$$

Clearly $2 U=U$. Let $W$ be the Banach space that is the completion of $Y$. Then we can consider $g$ to be a mapping from $X$ into $W$. Hence, by Corollaries 3.5 (when $p<1$ ) and 3.6 (when $p>1$ ) with $\delta=L_{1}=L_{2}=0, g$ is additive on $U$. Now it is enough to apply Proposition 3.8 analogously as before.

Remark 4.2. Note that examples (a), (c) and (e) in Remark 3.9 describe $\sigma$-ideals $\mathcal{I}$ satisfying condition (4.1).

The next two corollaries provide two further examples of simple applications of Theorem 3.1, which correspond to some results in [3,5,11-17,24] concerning the inhomogeneous Cauchy equation and the cocycle equation.

Corollary 4.3. Let $C, D: X \rightarrow X$ be additive, (3.1) and (3.2) be valid, and $G: U^{2} \rightarrow Y$ be such that $G\left(x_{0}, y_{0}\right) \neq 0$ for some $x_{0}, y_{0} \in U$ with $x_{0}+y_{0} \in U$. Assume that there exist positive reals $L$ and $p$ such that one of conditions (a) and (b) holds and

$$
\|G(x, y)\| \leq L\|C(x)-D(y)\|^{p} \quad x, y \in U, x+y \in U .
$$

Then the conditional functional equation

$$
g(x+y)=g(x)+g(y)+G(x, y) \quad x, y \in U, x+y \in U,
$$

has no solutions in the class of functions $g: U \rightarrow Y$. 
Proof. Let $g: U \rightarrow Y$ be a solution to (4.4). Then, in view of (4.3), (3.3) holds. Hence, by Theorem 3.1, $g$ is a solution to (1.1). This means that $G\left(x_{0}, y_{0}\right)=0$, which is a contradiction.

Corollary 4.4. Let $C, D: X \rightarrow X$ be additive, (3.1) and (3.2) be valid, and $G: X^{2} \rightarrow Y$ be a symmetric (i.e., $G(x, y)=G(y, x)$ for $x, y \in X$ ) solution to the cocycle functional equation

$$
G(x, y)+G(x+y, z)=G(x, y+z)+G(y, z) \quad x, y, z \in X .
$$

Assume that there exist positive reals $L$ and $p$ such that (4.3) and one of conditions (a) and (b) hold. Then $G(x, y)=0$ for $x, y \in U$ with $x+y \in U$.

Proof. Suppose that $G\left(x_{0}, y_{0}\right) \neq 0$ for some $x_{0}, y_{0} \in U$ with $x_{0}+y_{0} \in U$. It is well known that $G$ is coboundary (see [16] or [25]), i.e., there is $g: X \rightarrow Y$ with $G(x, y)=g(x+y)-g(x)-g(y)$ for $x, y \in X$. Hence $g$ is a solution to (4.4). This contradicts Corollary 4.3.

Analogous corollaries follow from Corollaries 3.5 and 3.6 with $\delta=L_{1}=$ $L_{2}=0$ and Propositions 2.1-2.3. For the convenience of the readers we end the paper with two of them, which are derived from Proposition 2.1.

Corollary 4.5. Let $(X,\langle\cdot \mid \cdot\rangle)$ be a real inner product space with $\operatorname{dim} X>1$ and $G: X^{2} \rightarrow Y$. Suppose that there are positive real numbers $p$ and $L$ with

$$
\|G(x, y)\| \leq L|\langle x \mid y\rangle|^{p} \quad x, y \in X .
$$

Then the following two statements are valid.

(A) If $p \neq 1$, then the functional equation

$$
g(x+y)=g(x)+g(y)+G(x, y)
$$

has a solution $g: X \rightarrow Y$ if and only if $G(x, y)=0$ for every $x, y \in X$.

(B) If $p=1$, then $g: X \rightarrow Y$ and $G$ satisfy (4.7) if and only if there exist additive $a: X \rightarrow Y$ and a vector $z_{0} \in Y$ such that

$$
g(x)=a(x)+\|x\|^{2} z_{0}, \quad G(x, y)=2\langle x \mid y\rangle z_{0} \quad x, y \in X .
$$

Proof. In the case of (A) it is enough to argue analogously as in the proof of Corollary 4.3, replacing Theorem 3.1 with Proposition 2.1.

So assume that $p=1$ and $g: X \rightarrow Y$ and $G$ satisfy (4.7). Then Proposition 2.1 implies that there exist additive $a: X \rightarrow Y$ and a vector $z_{0} \in Y$ such that $g(x)=a(x)+\|x\|^{2} z_{0}$ for $x \in X$. It is easy to check that this yields $G(x, y)=2\langle x \mid y\rangle z_{0}$ for $x, y \in X$. The converse is also easy to verify.

Corollary 4.6. Let $(X,\langle\cdot \mid \cdot\rangle)$ be a real inner product space with $\operatorname{dim} X>1$ and $G: X^{2} \rightarrow Y$ be symmetric. Suppose that there are positive real numbers $p$ and $L$ such that (4.6) holds. Then the following two statements are valid.

$1^{\circ}$ If $p \neq 1$, then $G$ is a solution to the cocycle functional Eq. (4.5) if and only if $G(x, y)=0$ for every $x, y \in X$. 
$2^{\circ}$ If $p=1$, then $G$ satisfies Eq. (4.5) if and only if there exists a vector $u_{0} \in Y$ such that $G(x, y)=\langle x \mid y\rangle u_{0}$ for $x, y \in X$.

Proof. If $p \neq 1$, then it is enough to argue analogously as in the proof of Corollary 4.4, replacing Corollary 4.3 with Corollary 4.5.

It remains to consider the case $p=1$. Then there is $g: X \rightarrow Y$ with $G(x, y)=g(x+y)-g(x)-g(y)$ for $x, y \in X$ (see [16] or [25]), whence $g$ is a solution to (4.7). Hence, by Corollary 4.5, there exist additive $a: X \rightarrow Y$ and a vector $z_{0} \in Y$ such that $g(x)=a(x)+\|x\|^{2} z_{0}$ for $x \in X$, whence $G(x, y)=2\langle x \mid y\rangle z_{0}$ for $x, y \in X$ and we take $u_{0}:=2 z_{0}$. The converse is easy to check.

Open Access. This article is distributed under the terms of the Creative Commons Attribution License which permits any use, distribution, and reproduction in any medium, provided the original author(s) and the source are credited.

\section{References}

[1] Aczél, J., Baker, J.A., Djoković, D.Ž., Kannappan, P., Radó, F.: Extensions of certain homomorphisms of subsemigroups to homorphisms of groups. Aequationes Math. 6, 263-271 (1971)

[2] Aoki, T.: On the stability of the linear transformation in Banach spaces. J. Math. Soc. Jpn. 2, 64-66 (1950)

[3] Borelli Forti, C.: Solutions of a non-homogeneous Cauchy equation. Rad. Mat. 5, 213222 (1989)

[4] Bourgin, D.G.: Approximately isometric and multiplicative transformations on continuous function rings. Duke Math. J. 16, 385-397 (1949)

[5] Brzdȩk, J.: On a generalization of the Cauchy functional equation. Aequationes Math. 46, 56-75 (1993)

[6] Brzdȩk, J.: On almost additive mappings. Bull. Aust. Math. Soc. 54, 281-290 (1996)

[7] Brzdęk, J., Pietrzyk, A.: A note on stability of the general linear equation. Aequationes Math. 75, 267-270 (2008)

[8] Ciepliński, K.: Applications of fixed point theorems to the Hyers-Ulam stability of functional equations - a survey. Ann. Funct. Anal. 3, 151-164 (2012)

[9] Ciepliński, K.: Stability of multi-additive mappings in $\beta$-Banach spaces. Nonlinear Anal. 75, 4205-4212 (2012)

[10] Czerwik S.: Functional equations and inequalities in several variables. World Scientific Publishing Co., River Edge (2002)

[11] Davison, T.M.K., Ebanks, B.: Cocycles on cancellative semigroups. Publ. Math. Debrecen 46, 137-147 (1995)

[12] Ebanks, B.: Generalized Cauchy difference functional equations. Aequationes Math. 70, 154-176 (2005)

[13] Ebanks, B.: Generalized Cauchy difference equations. II. Proc. Am. Math. Soc. 136, 3911-3919 (2008)

[14] Ebanks, B., Kannappan, P., Sahoo, P.K.: Cauchy differences that depend on the product of arguments. Glasnik Mat. 27(47), 251-261 (1992)

[15] Ebanks, B., Sahoo, P., Sander, W.: Characterizations of Information Measures. World Scientific, Singapore (1998)

[16] Erdös, J.: A remark on the paper "On some functional equations" by S. Kurepa. Glasnik Mat.-Fiz. Astronom. 14(2), 3-5 (1959)

[17] Fenyö, I., Forti, G.-L.: On the inhomogeneous Cauchy functional equation. Stochastica 5, 71-77 (1981) 
[18] Fisher, P., Słodkowski, Z.: Christensen zero sets and measurable convex functions. Proc. Am. Math. Soc. 79, 449-453 (1980)

[19] Fošner A.: On the generalized Hyers-Ulam stability of module left $(m ; n)$-derivations. Aequationes Math. doi:10.1007/s00010-012-0124-3

[20] Gajda, Z.: On stability of additive mappings. Int. J. Math. Math. Sci. 14, 431-434 (1991)

[21] Găvruţa, P.: A generalization of the Hyers-Ulam-Rassias stability of approximately additive mappings. J. Math. Anal. Appl. 184, 431-436 (1994)

[22] Hyers, D.H.: On the stability of the linear functional equation. Proc. Natl. Acad. Sci. USA 27, 222-224 (1941)

[23] Hyers, D.H., Isac, G., Rassias Th.M.: Stability of Functional Equations in Several Variables. Birkhäuser, Boston (1998)

[24] Járai, A., Maksa, Gy., Páles, Zs.: On Cauchy-differences that are also quasisums. Publ. Math. Debrecen 65, 381-398 (2004)

[25] Jessen, B., Karpf, J., Thorup, A.: Some functional equations in groups and rings. Math. Scand. 22, 257-265 (1968)

[26] Jung, S.-M.: Hyers-Ulam Stability of Functional Equations in Mathematical Analysis. Hadronic Press, Palm Harbor (2001)

[27] Jung S.-M.: Hyers-Ulam-Rassias Stability of Functional Equations in Nonlinear Analysis. Springer Optimization and Its Applications, vol. 48. Springer, New York (2011)

[28] Kuczma, M.: An Introduction to the Theory of Functional Equations and Inequalities. Państwowe Wydawnictwo Naukowe \& Uniwersytet Śląski, Warszawa (1985)

[29] Maksa, Gy., Páles, Zs.: Hyperstability of a class of linear functional equations. Acta Math. Acad. Paedag. Nyìregyháziensis 17, 107-112 (2001)

[30] Moszner, Z.: On the stability of functional equations. Aequationes Math. 77, 33-88 (2009)

[31] Piszczek, M.: The properties of functional inclusions and Hyers-Ulam stability. Aequationes Math. doi:10.1007/s00010-012-0119-0

[32] Rassias, Th.M.: On the stability of the linear mapping in Banach spaces. Proc. Am. Math. Soc. 72, 297-300 (1978)

[33] Rassias, Th.M.: On a modified Hyers-Ulam sequence. J. Math. Anal. Appl. 158, 106113 (1991)

[34] Rassias, Th.M., Isac, G.: Functional inequalities for approximately additive mappings. In: Rassias, Th.M., Tabor, J. (eds.) Stability of Mappings of Hyers-Ulam Type, pp. 117-125. Hadronic Press, Palm Harbor (1994)

[35] Rätz, J.: On orthogonally additive mappings. Aequationes Math. 28, 35-49 (1985)

[36] Sahoo, P.K., Kannappan, P.: Introduction to Functional Equations. CRC Press, Boca Raton (2011)

[37] Skof, F.: On the stability of functional equations on a restricted domain and related topics. In: Rassias, Th.M., Tabor, J. (eds.) Stability of Mappings of Hyers-Ulam Type, pp. 141-151. Hadronic Press, Palm Harbor (1994)

[38] Szabó, Gy.: A conditional Cauchy equation on normed spaces. Publ. Math. Debrecen 42, 265-271 (1993)

[39] Szabó, Gy.: Isosceles orthogonally additive mappings and inner product spaces. Publ. Math. Debrecen 46, 373-384 (1995)

Janusz Brzdęk

Department of Mathematics

Pedagogical University

Podchorążych 2

30-084 Kraków, Poland

e-mail: jbrzdek@up.krakow.pl

Received: July 24, 2012 